\title{
UDC 634.8
}

\section{SCOPUS CODE 1101}

https://doi.org/10.36073/1512-0996-2021-2-29-37

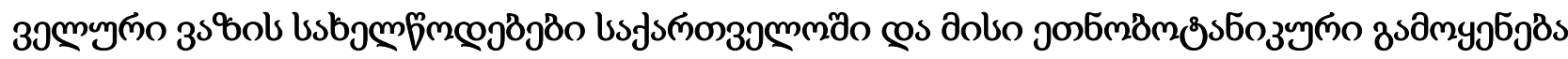

\author{
әјбаэмо \\ зозомедазомо

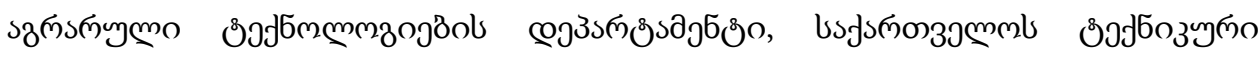

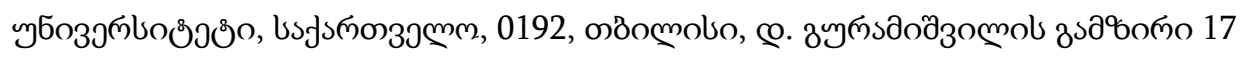 E-mail: shengelikikilashvili@gmail.com

\section{๓วงว}

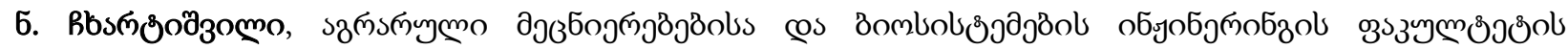

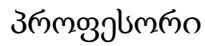

E-mail: n_chkhartishvili@gtu.ge

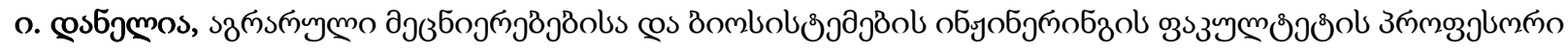

E-mail: i.danelia@gtu.ge

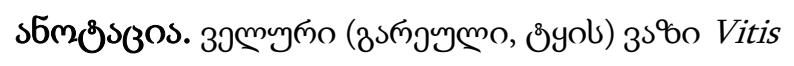
vinifera subsp. sylvestris (C.C.Gmel.) Hegi bsjs(nom39-

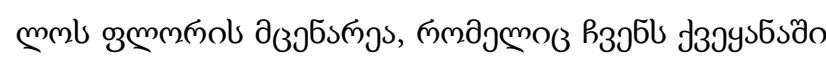

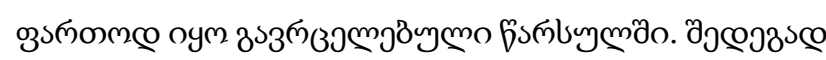

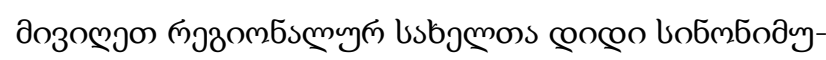

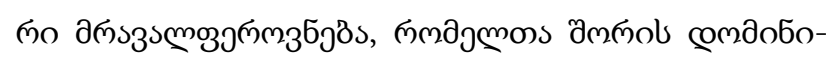

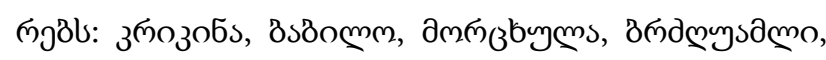

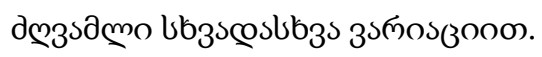

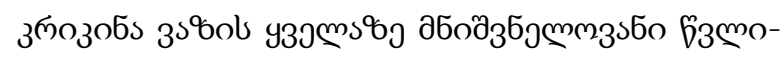

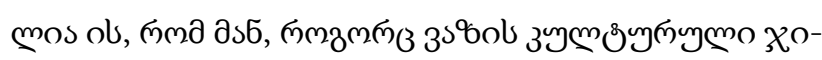

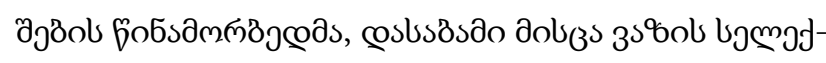

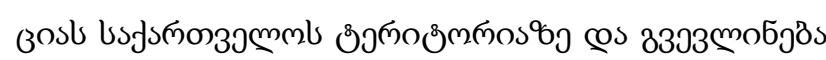

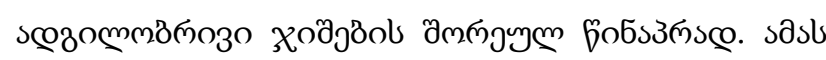

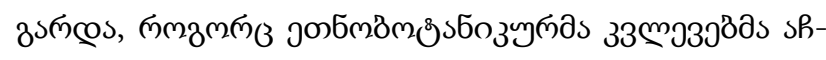

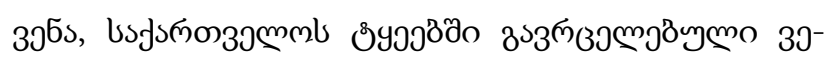

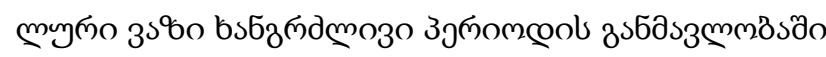

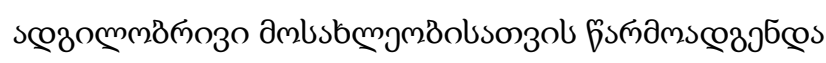

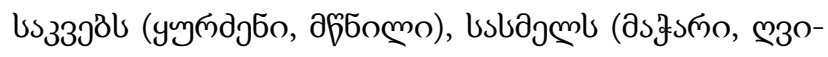

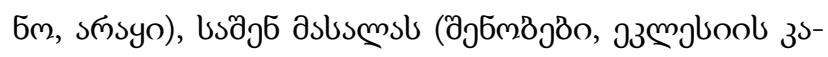

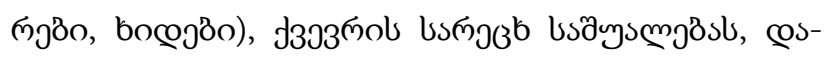

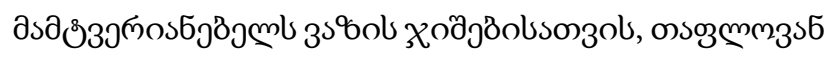
а

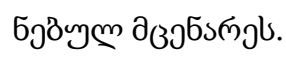

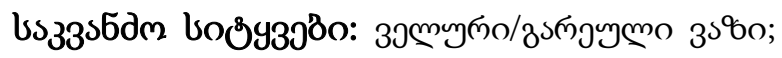

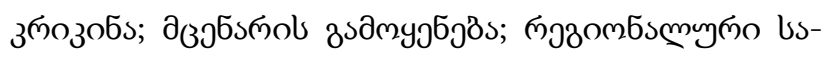
ьวмэว̊̊.

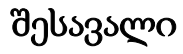

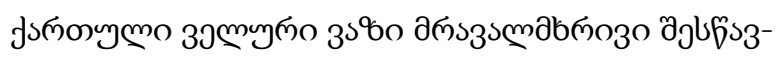

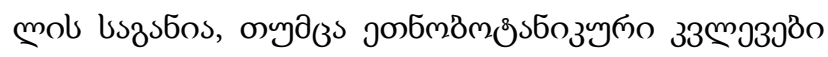

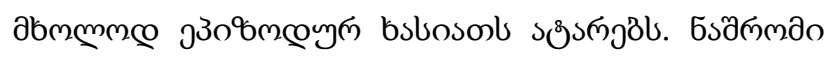

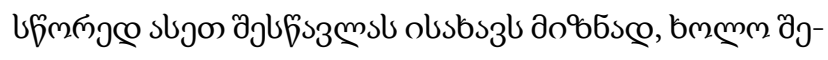

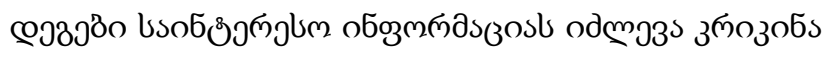




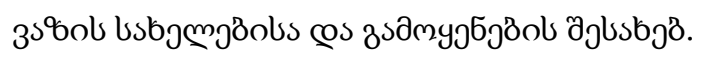

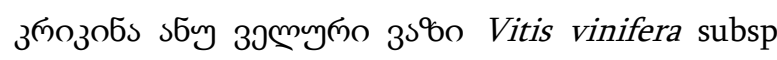

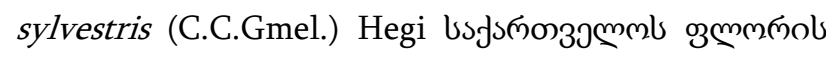

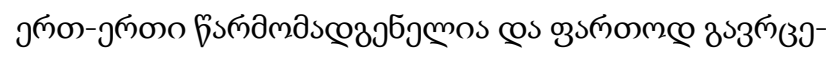

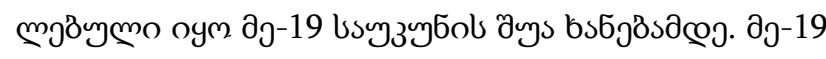

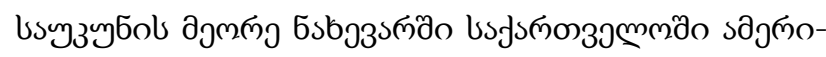

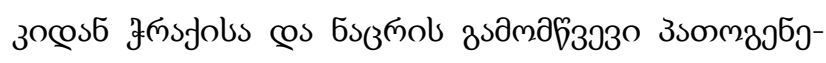

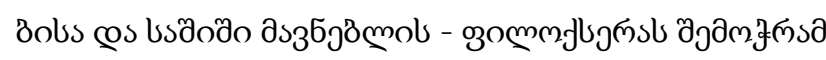

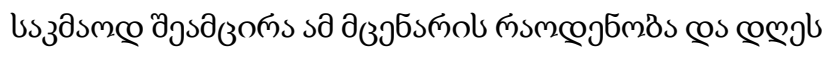

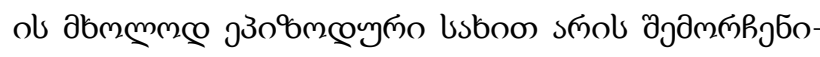

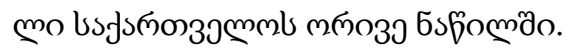

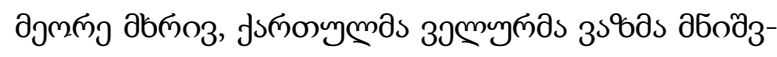

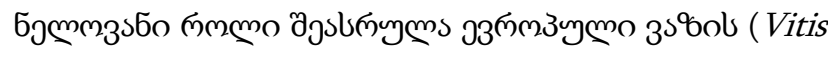

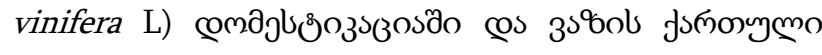

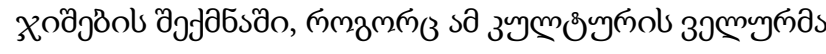

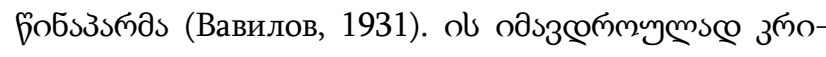

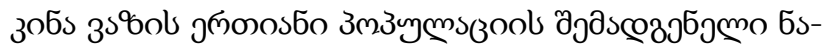

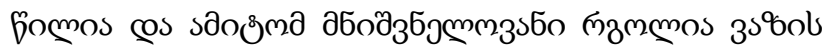

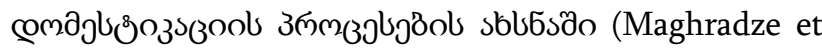

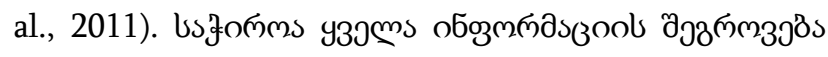

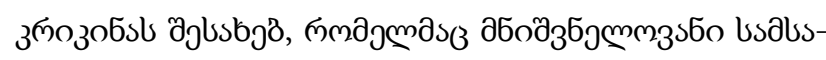

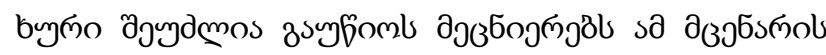

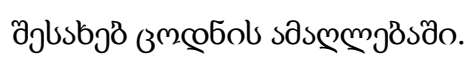

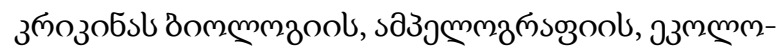

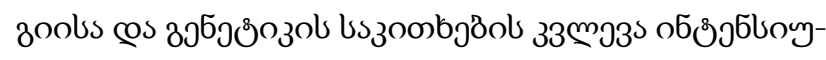

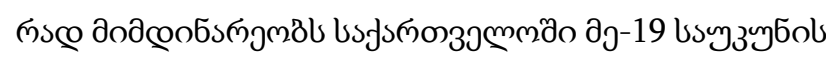

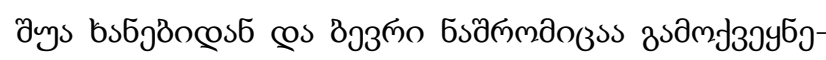

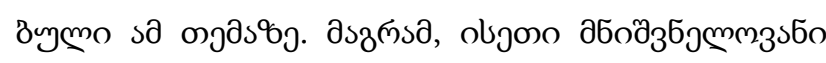

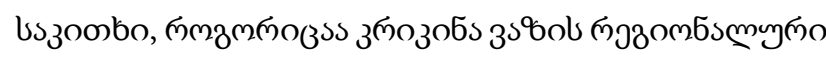

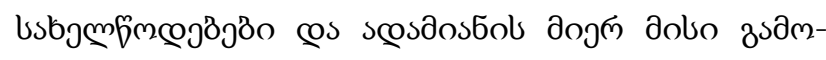

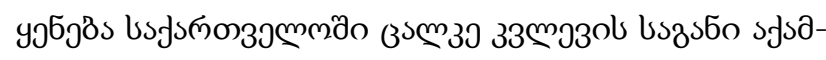

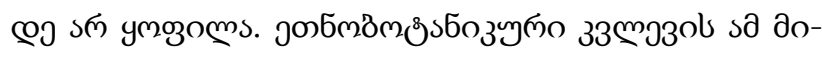

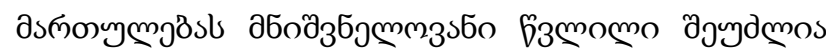

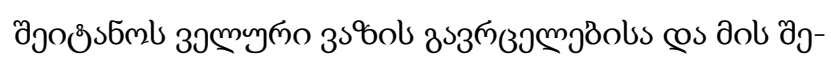

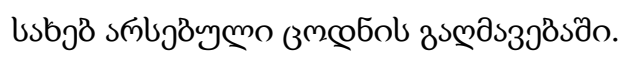

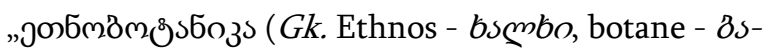

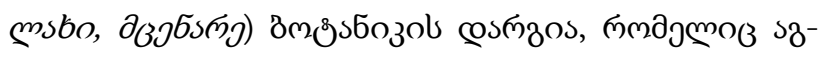

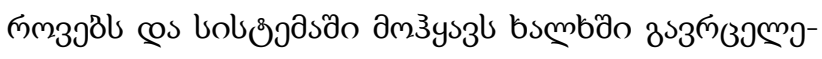

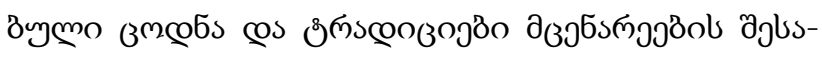

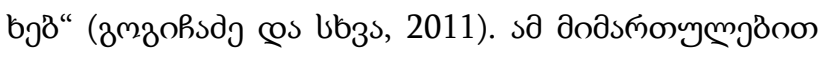

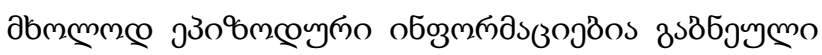

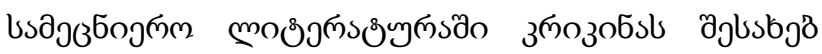
(аsмñ

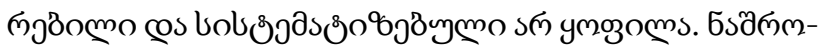

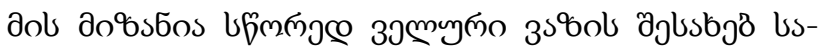

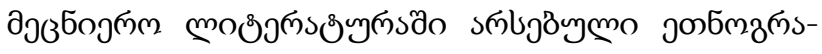

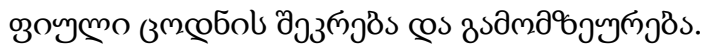

\section{donoossco 6sfomo}

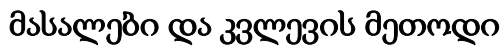

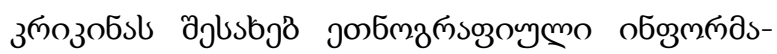

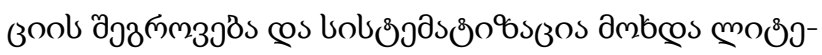

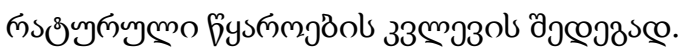

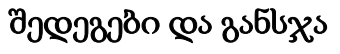

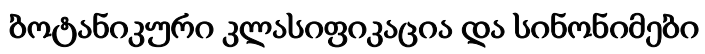

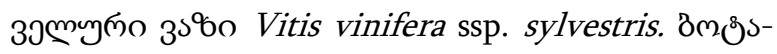

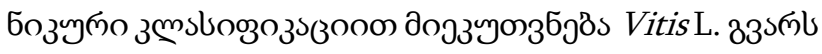

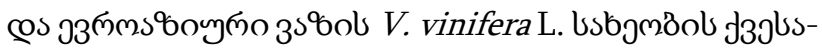

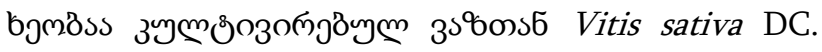

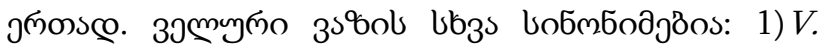
sylvestris C. C. Gmel; 2) V. vinifera sylvestris (C. C. Gmel.) DC; 3) V. vinifera L. subsp sylvestris (C.C. Gmel) Hegi; 4) Vitis vinifera ssp. sylvestris (Gmelin) Hegi; 5) V. vinifera silvestris Beck. 


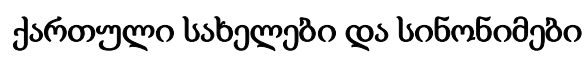

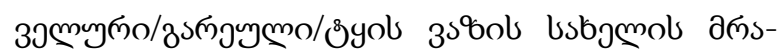

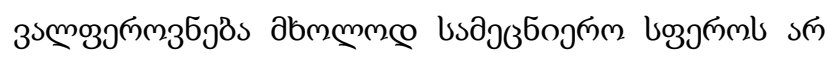

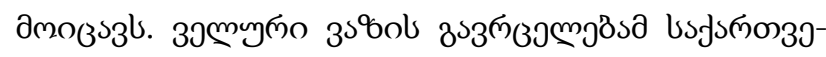

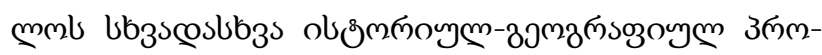
зоб

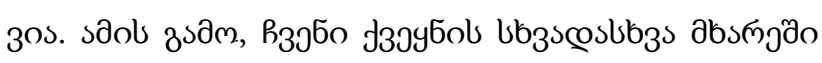

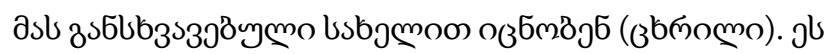

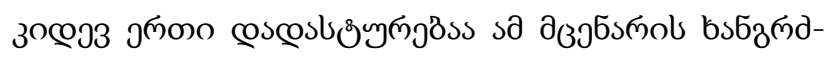

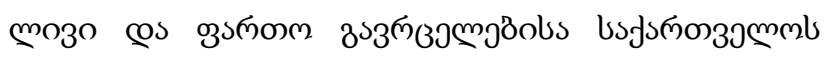

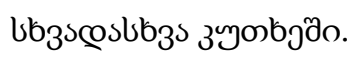

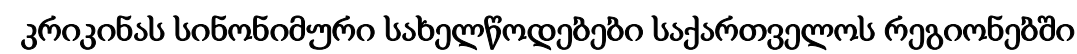

\begin{tabular}{|c|c|c|}
\hline costusbjmjas & absmృ, cosmajo̊o & కЗతిmณం \\
\hline 8sonmm & допуч⿻上丨о & 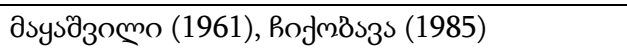 \\
\hline 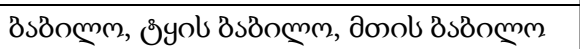 & j১ங்оmo & дsysəzomo(1961), slssomos6o (1978) \\
\hline впдмэуздмо & 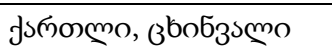 & sbsoos6o (1978) \\
\hline 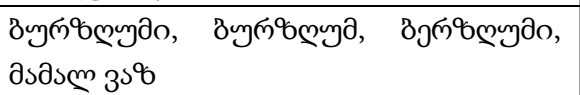 & usobgomm & asysəి3оmo (1961), susomos6o (1978) \\
\hline вйдамудо & bsoбzомма, зььјоо & susons6o (1978) \\
\hline 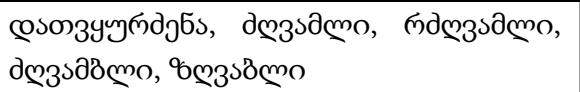 & msłs & 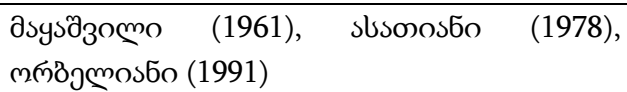 \\
\hline 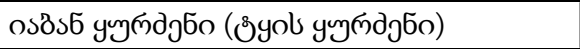 & 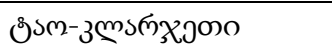 & Rsल̆33о56o (2011) \\
\hline उsłozo & одј๓дюо & susonos6o (1978) \\
\hline зоп̈зббs & 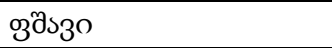 & asysð̃omo (1961) \\
\hline 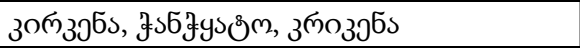 & joboyo & дsysə̋omo (1961), susoos6o (1978) \\
\hline зопुзобы & 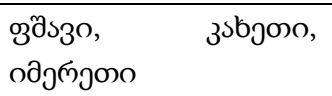 & 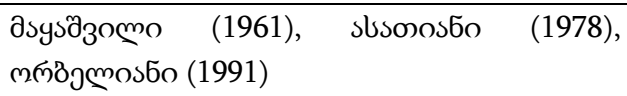 \\
\hline зॅозобs & 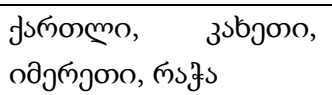 & susonos6o (1978), Rojm8̇s3s (1986) \\
\hline 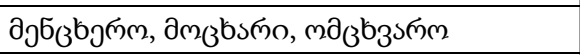 & mghbyда & asysð̄omo (1961) \\
\hline дmn̋buyms & ву丨लn & asysə̄ąomo (1961), susoos6o (1978) \\
\hline 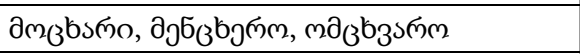 & mjßbуyдо & susonos6o (1978) \\
\hline 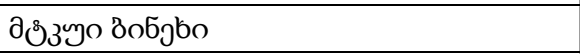 & 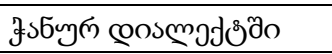 & 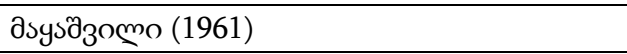 \\
\hline 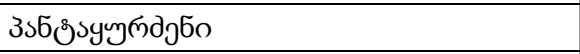 & słsms & susonos6o (1978) \\
\hline 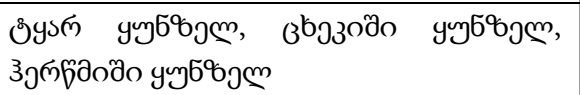 & ৮зsбјо & 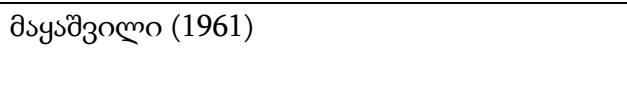 \\
\hline yluym & 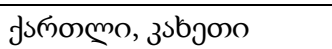 & susoos6o (1978), hojmß̊şక (1986) \\
\hline 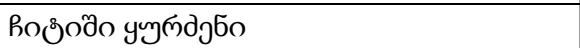 & иьдэвґомм & asysð̃omo (1961) \\
\hline 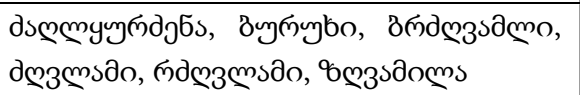 & одјґృю & дsysə̃zоmо (1961), susonos6o (1978) \\
\hline 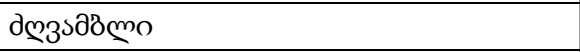 & ๓słs, mfibyao & susonos6o (1978) \\
\hline 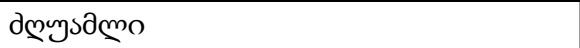 & - & 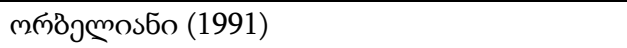 \\
\hline 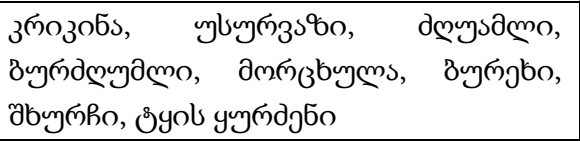 & 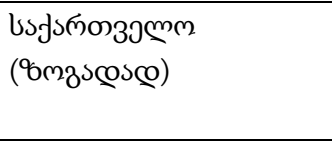 & 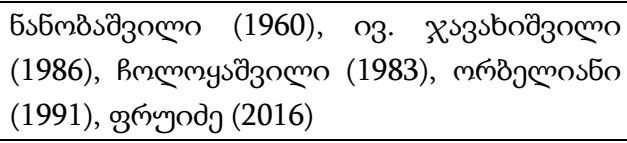 \\
\hline
\end{tabular}




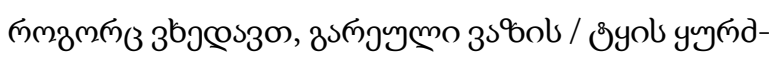

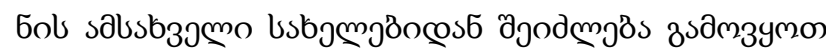

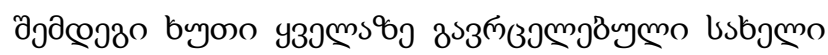

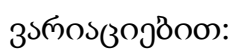

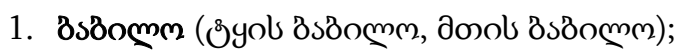

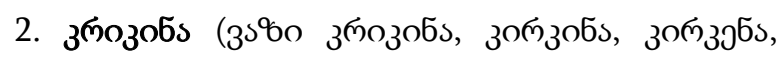
उलо3эгs);

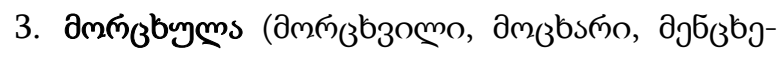

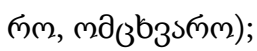

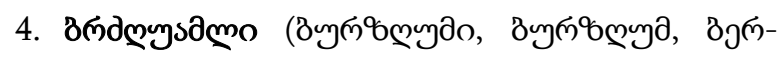

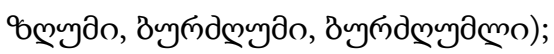

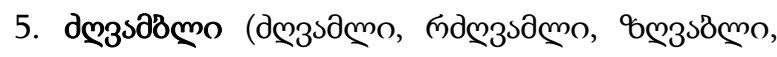

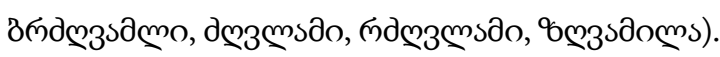

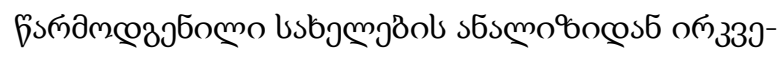

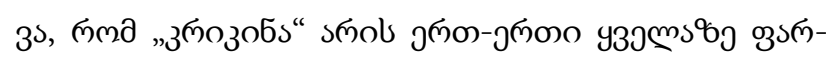

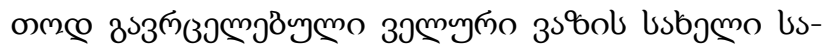

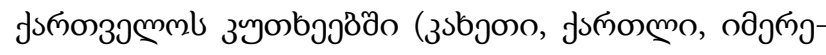

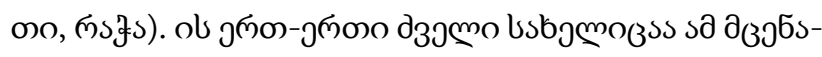

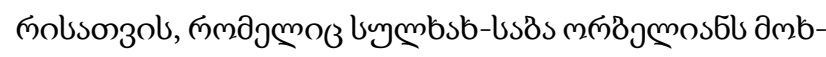

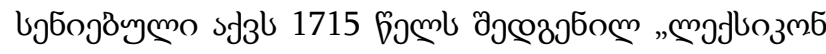

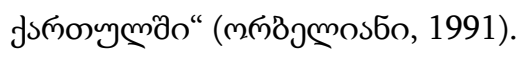

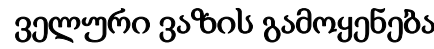

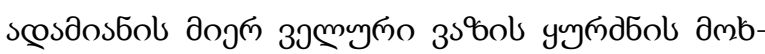

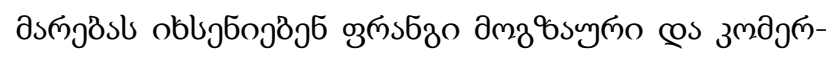

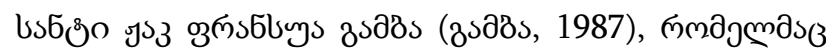

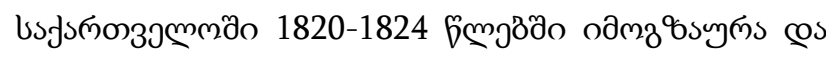

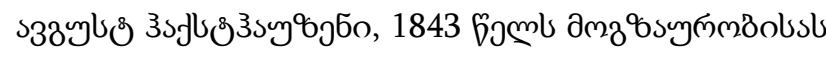

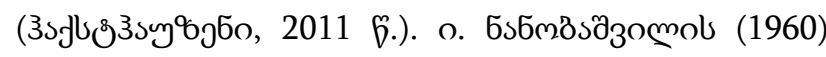

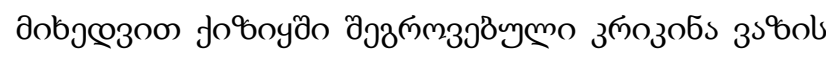

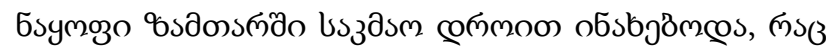

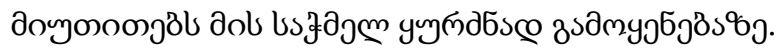

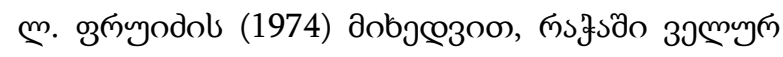

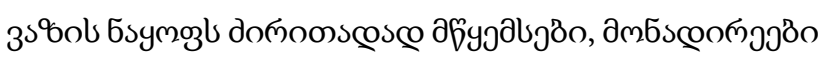

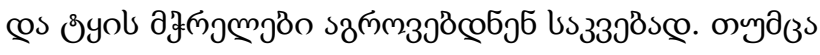

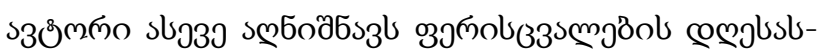

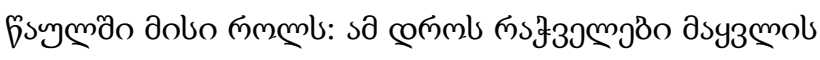

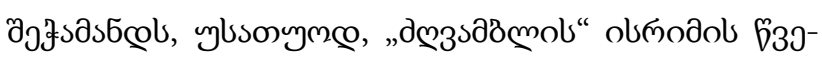

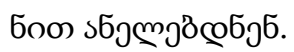

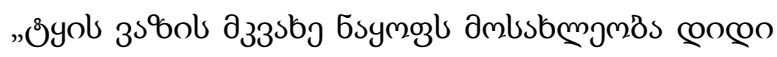

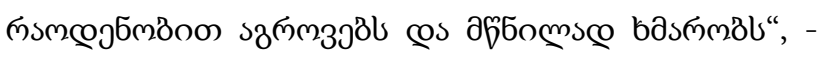

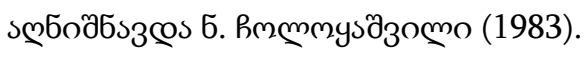

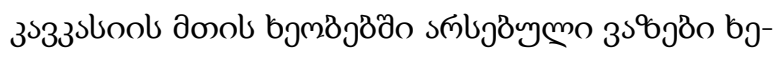

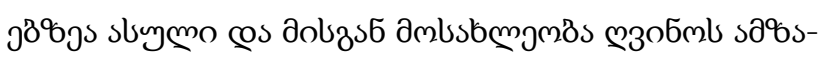

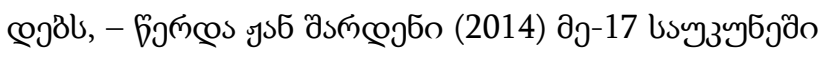

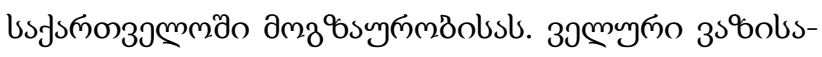
3

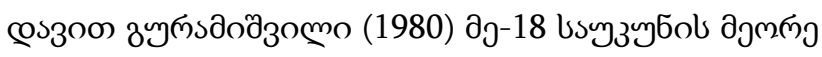

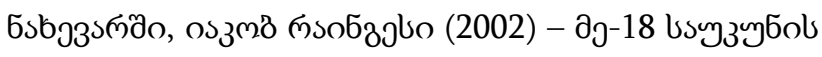

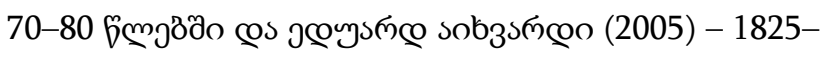
1826 белэвةо.

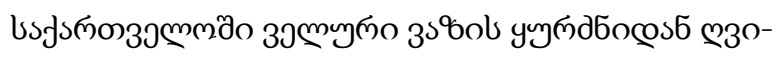

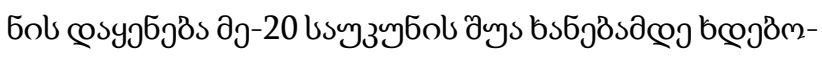

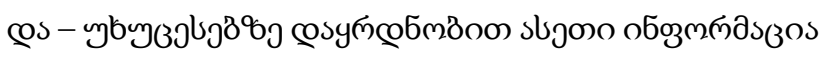

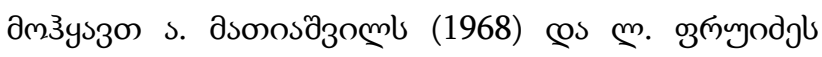

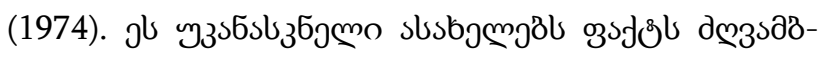

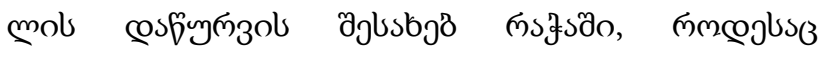

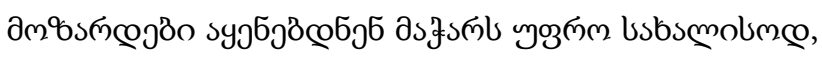

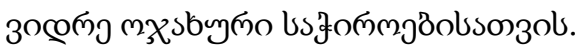

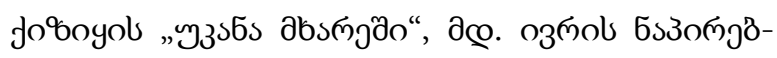

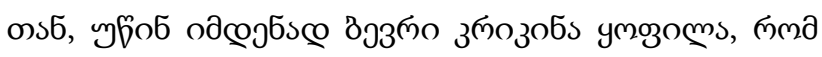

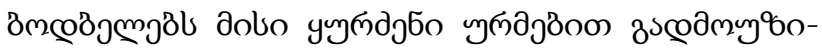

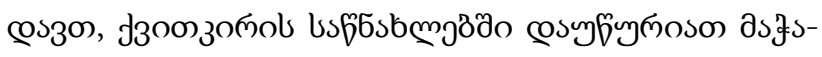

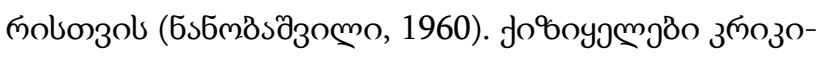




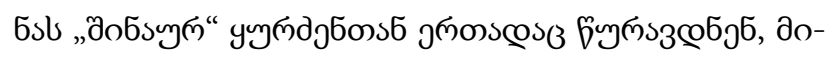

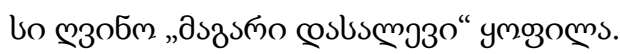

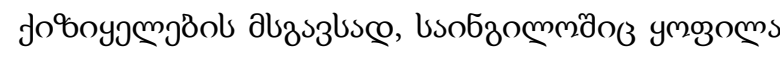
з зй

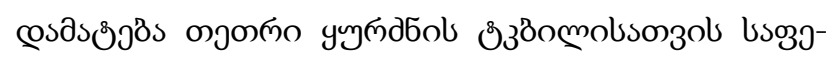

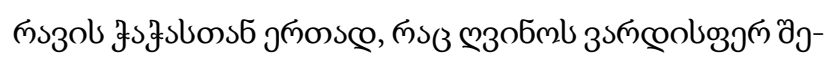

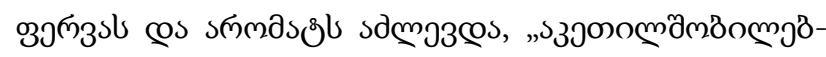

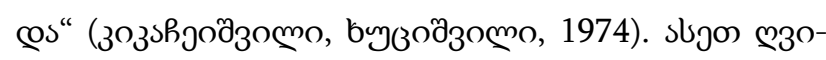

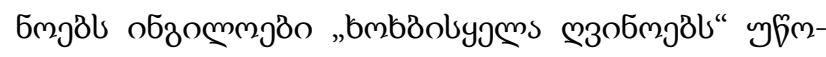

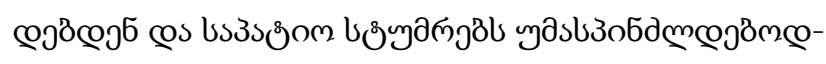

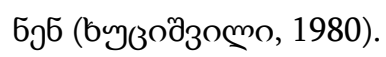

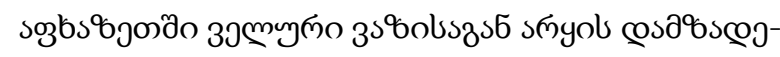

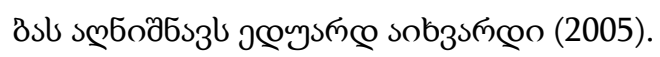

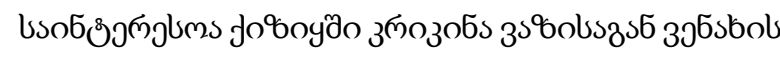

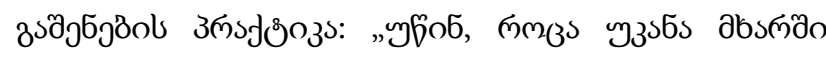
3

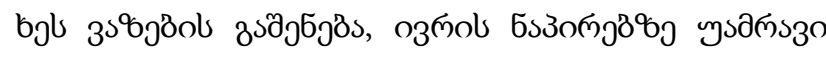

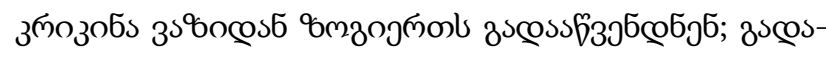

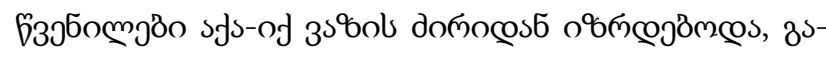

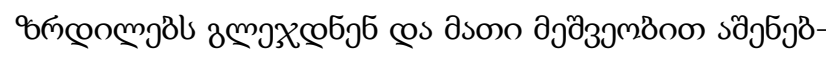

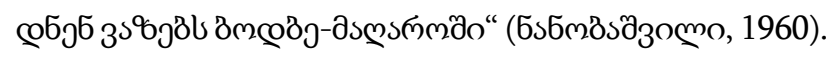

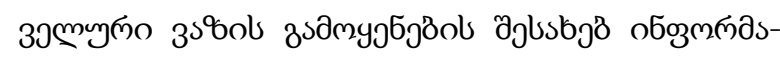

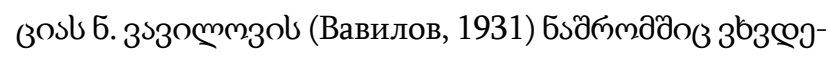

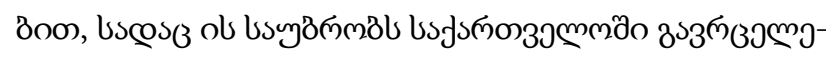

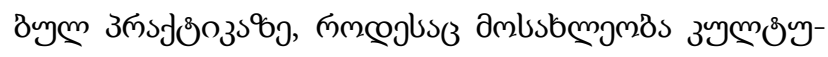
пилм 3

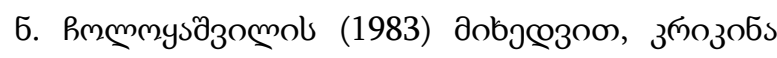

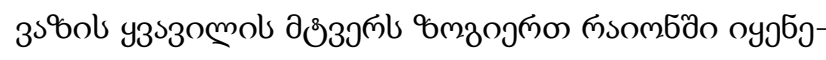

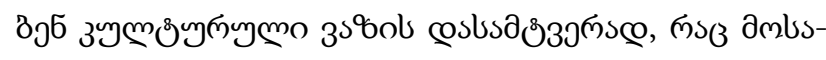

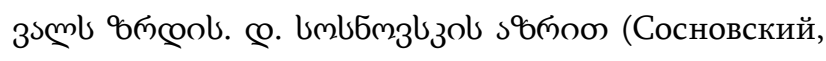

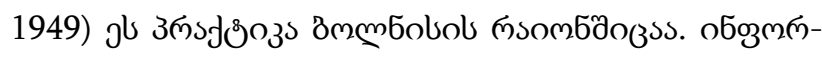

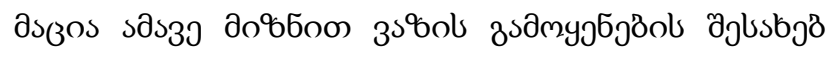

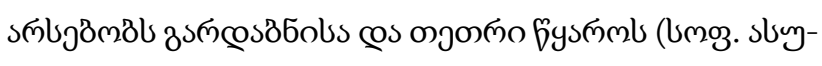

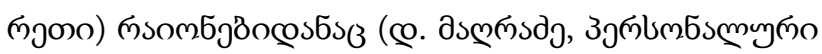
обозмпаз (зоз).

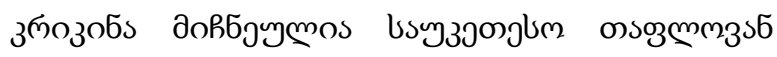

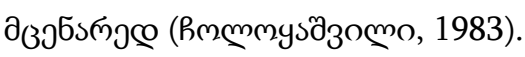

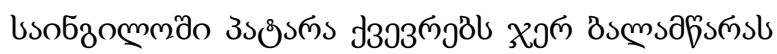

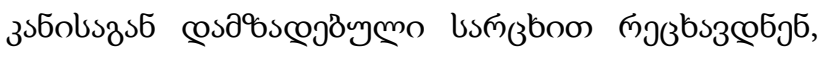

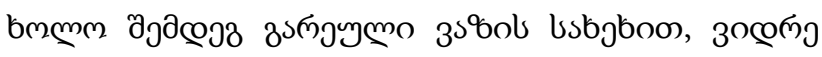

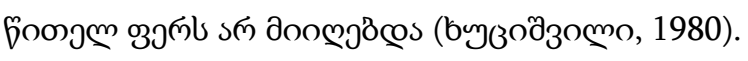

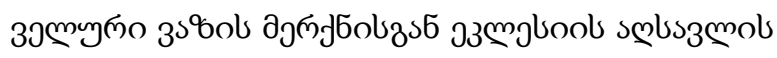

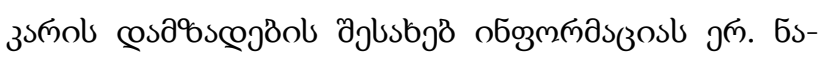

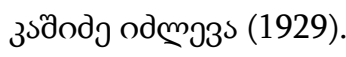

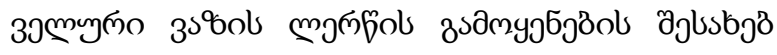

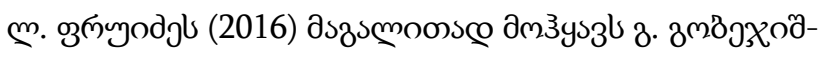

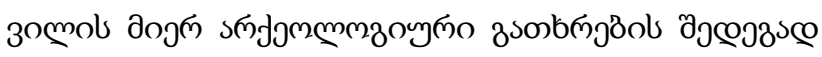

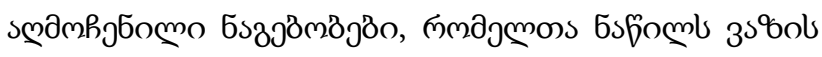

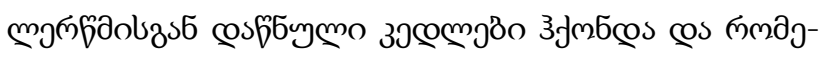

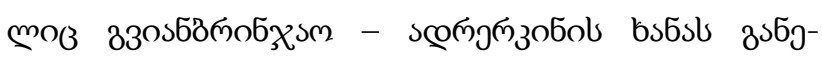

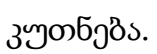

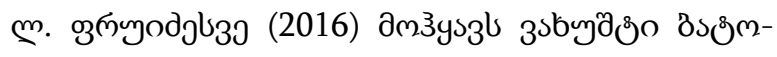

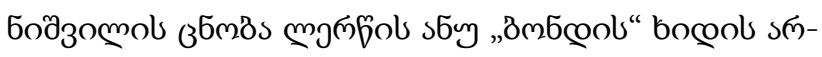

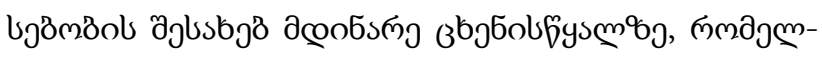

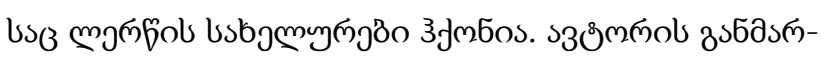

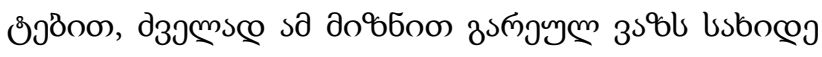

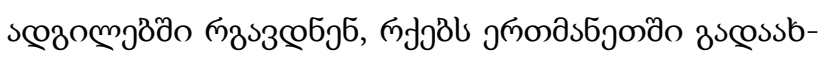

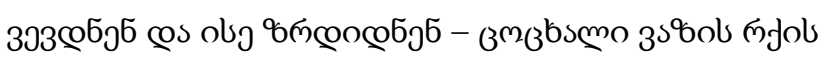

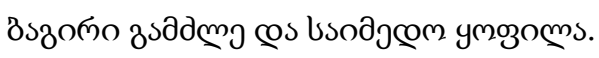

\section{$\cos 333^{6 s}$}

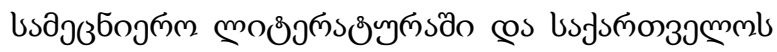

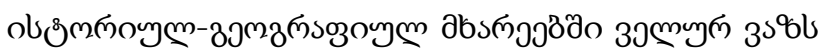

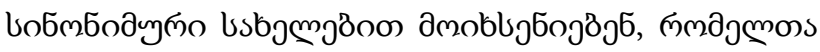




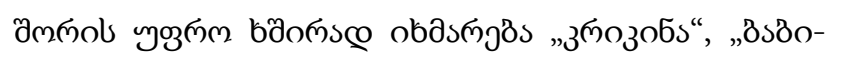

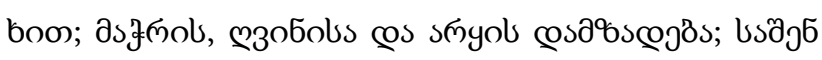

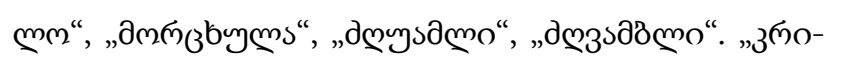

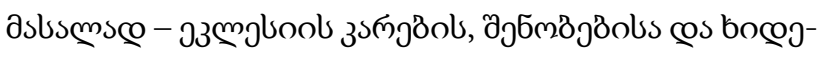

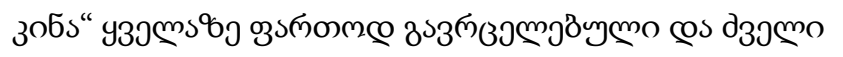

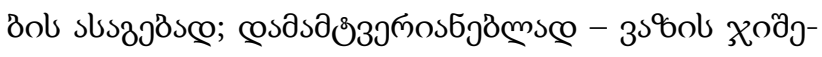

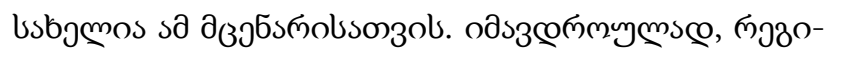

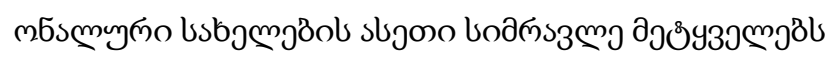

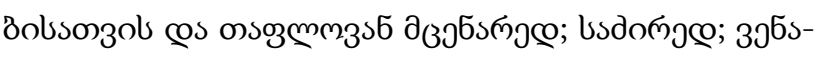

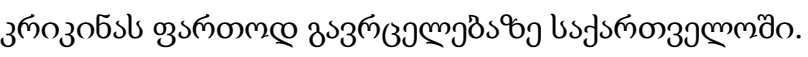

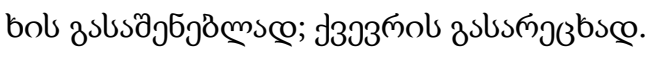

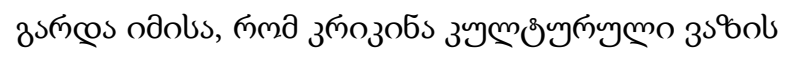

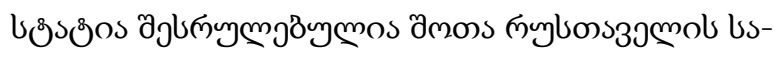

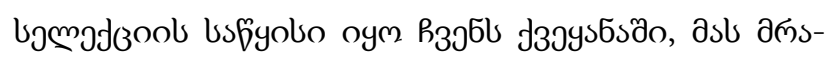

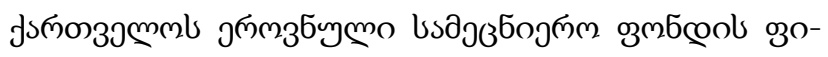

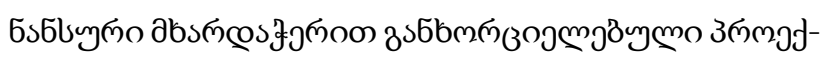

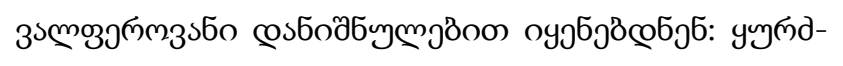

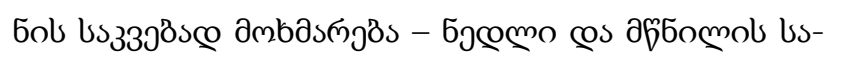

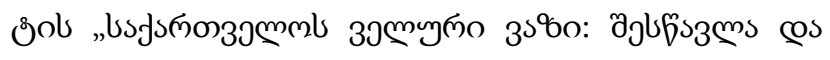

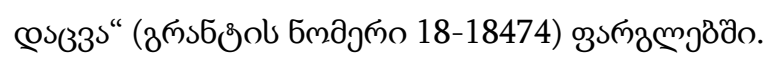

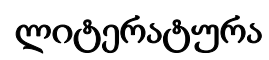

1. Aikhvard E. About Georgia (first third of the XIX century) Translated from German into Georgian by G. Gelashvili. 2005 Tbilisi. pp. 332. (In Georgian).

2. Asatiani L. Vocabulary related to vine culture in Georgia. 1978 Tbilisi. pp. 86-91 (In Georgian).

3. Charkviani A., Maglaperidze L., Khuchua L. Wild grapevine of Tao-Klarjeti. Student expedition 'Tao-Klarjeti 2011'. Collection of the papers. "Free University" Publisher. 2011. Tbilisi. pp. 60-76. (In Georgian).

4. Chikobava A. (Editor). Dictionary of Georgian Language. In- Volume. Institute of Languges of the Georgian Academy of Sciences. Georgian Soviet Enciclopedia 1986. Tbilisi. pp. 270 (In Georgian).

5. Cholokashvili N. Family Vitaceae. Flora of Georgia. 2nd edition. Publishing House "Ganatleba". 1983. Tbilisi. Vol.8. pp. 274-278 (In Georgian).

6. Chardin J. Travel to Georgia. Translated by V. Barnov. 2014. Tbilisi. pp. 36-58 (In Georgian).

7. Gamba. J.F.. Travel to Transcaucasia. Translated from French into Georgian M. Mgaloblisvil. 1987. Tbilisi. pp. 225 (In Georgian).

8. Gogichadze G., Kandelaki G., Gogichadze T. Dictionary: biological and medical terms and concepts. Publishing House „Meridian”. 2011. Tbilisi. pp. 442. (In Georgian).

9. Guramishvili, D. Davitiani (Book of David), Poem, Publishing houses "Khelovneba", Tbilisi, Georgia and "Mistectvo", Kiev, Ukraine, 1980. pp 85 (in Georgian).

10. Huxthausen A. About Georgia (first half of the XIX century). Translated from German by G. Gelashvili. 2011 Tbilisi. pp. 40-226 (In Georgian).

11. Javakhishvili Iv. Economic History of Georgia. The second Book. Selected scientific works in twelve volumes. Volume 5. Tbilisi. 1986. pp. 308-311 (In Georgian).

12. Kikacheishvili R., Khutsishvili I. Peculiarities of wine making and other grape products in Saingilo. Scientific works of the institute of Horticulture, Viticulture and Oenology.- Vol 13. 1974. Tbilisi. pp.240 (In Georgian).

13. Khutsishvili I. Viticulture in Saingilo. 1980. Tbilisi. pp. 19 (In Georgian). 
14. Magradze D. Krikina (wild) grapevine in Georgia. Veluri Buneba. 2020. Tbilisi. pp. $46-49$ (In Georgian).

15. Maghradze D., Failla O., Imazio S., Becilieri R., Chipashvili R., Rubio O. R., Quattrini E., This P., Scienza A. Wild grapevine in Georgia. Origini della Viticoltura. 2011. Pg 183-107 (In English).

16. Makashvili A. Botanical Dictionary. Plant names. 1961. Tbilisi. pp. 260 (In Georgian).

17. Matiashvili A. Vine and wine in Davitiani. Dzeglis Megobari. Vol. 16. 1968. Tbilisi. pp. 69 (In Georgian).

18. Nakashidze E. Viticulture and winemaking in Guria-Samegrelo, Adjara and Abkhazia. Publishing House "Sakhelgami". 1929. Tbilisi. pp. 61 (In Georgian).

19. Nanobashvili I. Old vine culture in Qiziki. 1960. Tbilisi. pp. 47-52 (In Georgian).

20. Orbeliani S.S. Georgian Dictionary. In two volumes. Edited by I. Abuladze. Publishing House. "Merani" 1991. www.nplg.gov.ge/gwdict/index.php?a=term\&d=8\&t=21101 (In Georgian).

21. Pruidze L. Viticulture and winemaking in Georgia. The first book: Racha. 1974. Tbilisi. pp. 47-51 (In Georgian).

22. Pruidze L. History of viticulture and winemaking of Georgia. In two volums. Vol.2 Viticulture. 2016. Tbilisi. pp. 38-64 (In Georgian).

23. Reingens I. Travel to Georgia. Translated from German into Georgian by G. Gelashvili. 2002 Tbilisi. pp. 131157 (In Georgian).

24. Sosnovskii D.I. Grapes - Vitaceae Lindl.: Flora of the USSR. In 30 volumes. Vol. 14. Ed. in chief Komarov V.L. Publishing house of the Academy of Sciences of the USSR. Moscow-Leningrad, 1949. pp. 700 (In Russian).

25. Vavilov N.I. Wild relatives of fruit trees in the Asian part of the USSR and the Caucasus and the problem of the origin of fruit trees. Works on applied botany, genetics and breeding, v. 36. No. 3. [The edition was used: Academician NI Vavilov - Selected works in five volumes. Volume II. Publishing house of the USSR Academy of Sciences. 1931 Moscow-Leningrad . pp. 343-361 (In Russian). 


\section{UDC 634.8}

SCOPUS CODE 1101

https://doi.org/10.36073/1512-0996-2021-2-29-37

\section{Local Names of Wild Grapevine in Georgia and Its Ethnobotanical Use}

Shengeli Kikilashvili Department of Agricultural Technologies, Georgian Technical University, Georgia, 0192, Tbilisi, 17 D. Guramishvili ave

E-mail: shengelikikilashvili@gmail.com

\section{Reviewers:}

N. Chkhartishvili, Professor, Faculty of Agrarian Sciences and Biosystems Engineering, GTU

E-mail: n_chkhartishvili@gtu.ge

I. Danelia, Professor, Faculty of Agrarian Sciences and Biosystems Engineering, GTU

E-mail: i.danelia@gtu.ge

Abstract. Wild grapevine Vitis vinifera subsp. sylvestris (C.C.Gmel.) Hegi is a typical plant of Georgian flora, spread in our country. Consequently, a numerous number of local synonym names of this plant are available in different historical-ethnographic provinces of Georgia. Among these names 'Krikina', 'Babilo', 'Mortskhula', 'Brdzghuamli' and 'Dzghvamli' are more spread, having different spelling variations.

The great contrubution of wild grape is that it, as an ancestor of cultivated grapevine, made the basis of grape breeding in Georgia and is a distant relative of our varieties. Besides, as it was demonstrated by the ethnobotanical research, due to long period present in the forests of Georgia the local people used this plant as a food (grape, pickles), a beverage (young fermented wine 'Machari', wine, grappa), a construction material (buildings, door for a church, breedge), a tool for washing Qvevri, a pollinator for grape cultivars, a honey plant, a rootstock, a planting material for vineyards.

Key words: 'Krikina'; plant usage; pollinator; regional names; rootstock; wild grapevine; wine. 


\title{
UDC 634.8
}

SCOPUS CODE 1101

https://doi.org/10.36073/1512-0996-2021-2-29-37

\section{Местные названия дикого винограда в Грузии и его этноботаническое использование}

\author{
Шенгели Департамент Аграрных технолоогий, Грузинский технический университет,

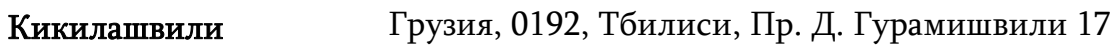 \\ E-mail: shengelikikilashvili@gmail.com
}

\section{Рецензенты:}

Н.Чхартишвили, профессор факультета аграрных наук и биосистем инженеринга ГТУ n_chkhartishvili@gtu.ge

И. Данелия, профессор факультета аграрных наук и биосистем инженеринга ГТУ

E-mail: i. danelia@gtu.ge

Аннотация. Дикий виноград Vitis vinifera subsp. sylvestris (C.C.Gmel.) Hegi - типичное растение грузинской флоры, распространенное в нашей стране. Следовательно, в разных историко-географических провинциях Грузии имеется большое количество местных синонимов этого растения. Среди них более распространены «Крикина», «Бабило», «Морцхула», «Брдзгуамли» и «Дзгвамли», имеющие разные варианты орфографии.

Большой вклад дикого винограда заключается в том, что он, как предок культивируемой виноградной лозы, лег в основу генофонда грузинских сортов и является дальним родственником наших сортов. Кроме того, как показали этноботанические исследования, благодаря длительному распространению в лесах Грузии, местное население употребляло его как пищу (виноград, соленья), напиток (молодое ферментированное вино «Мачари», вино, водка), строительный материал (постройки, церковные двери, мосты), средство для мытья квеври, опылитель для сортов винограда, медонос, подвой, посадочный материал для виноградников.

Ключевые слова: вино; дикий виноград; использование растений; «Крикина»; опылитель; подвой; региональные названия.

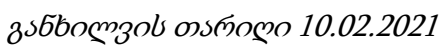

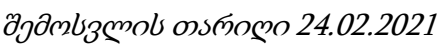

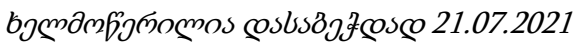

